\title{
A CFD ANALYSIS IN SOLIDWORKS FLOW SIMULATION FOR TWO MIXING FLUIDS WITH DIFFERENT TEMPERATURES IN NOZZLES
}

\author{
OLARU IONEL $\mathbf{1}^{*}$ \\ I "Vasile Alecsandri" University of Bacau, Calea Marasesti 157, Bacau, 600115, Romania
}

\begin{abstract}
The heat can have a direct impact on the mechanical elements by creating deformations and by causing the induction of additional stress in them. In designing of the heat exchangers or for the electronic components, the temperature changes for structural analysis as well as structural performance of thermal impact for the entire element must be analyzed. The study from this paper proposes an analysis and a simulation of flow through the convergent-divergent nozzle type to optimize the inlet of warm fluid to have minimum impact on the nozzle walls This analysis will be performed with a computer program specialized in complex analysis of Computational Fluid Dynamics (CFD), which will also take into account the fluid temperature and its influence throughout the system.
\end{abstract}

Keywords: flow, simulation, temperatures, fluid, nozzle

\section{INTRODUCTION}

In the case of different types of engineering systems, it's particularly important to understand how the metallic structure behaves in contact with the fluid flowing inside or outside. The properties of the temperature-dependent material, the temperature gradient and thermally induced deformation by the fluids with they react are important in case of designing the successful products [1].

With the simulations with SolidWorks 2019 Fluid Flow Analysis, designers can perform stress analyzes to determine the response of different elements under the action of forces, pressures or temperatures, to perform a multi-physical analysis. In the field of heat exchangers and not only it's important to understand all these phenomena and to be well implemented [2].

Thermal effects in engineering are often determined by a series of complex physical processes, such as: heat conducting, heat convection, conjugate with heat transfer between liquids, solid materials and radiation. If we also consider the multitude of complex geometries of the different elements, the need for accurate prediction of CFD analysis is essential to ensure the performance of the product, this can be extremely difficult to estimate or calculate manually [3].

This paper aims to study a type of nozzle in which fluids of different temperatures are introduced through two inlets of different diameters, with different angles. CFD analysis wants to establish an optimal ratio between the direction of the fluid jets, the rough action of the walls of the nozzle, as well as the influence of the temperature on these walls [4].

\footnotetext{
* Corresponding author, email: ionelo@ub.ro

(C) 2020 Alma Mater Publishing House
} 
Using this type of convergent divergent nozzle can be achieved by the computer simulation to analyze velocity within this system. To achieve the simulation were used as working fluids such as water and analysis of mixed fluid with two temperatures and pressures [5].

\section{SIMULATIONS SETUP}

This study starts from a reference nozzle geometry, nozzle geometry of this are shown in Figure 1. The diameter of the cold fluid inlet area is $10 \mathrm{~mm}$ and $2 \mathrm{~mm}$ for the hot fluid, the total pressure and the temperature differ in different areas of the nozzle. The mixing fluid studied in the paper is water, which is a common and cheap fluid.

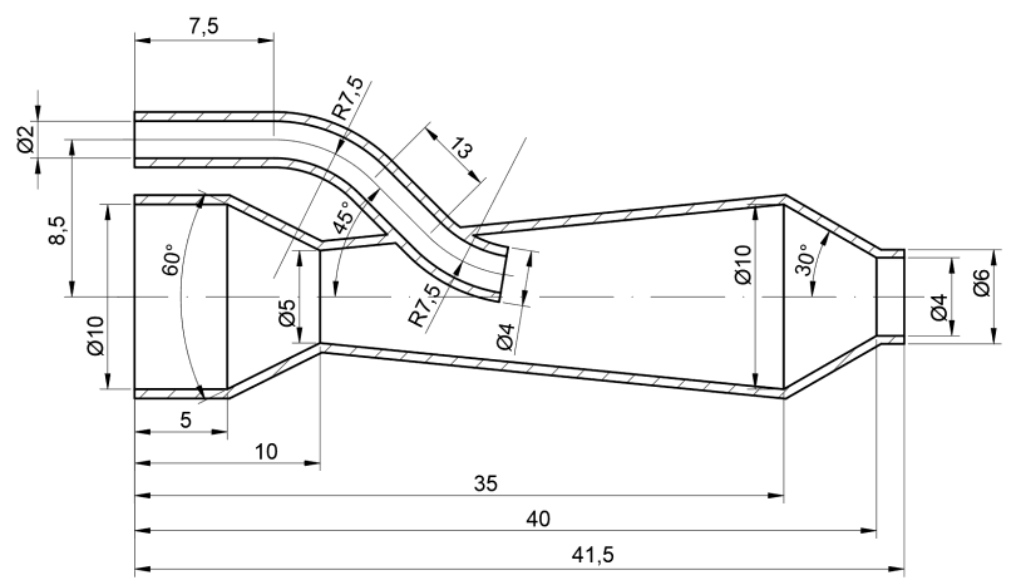

Fig. 1. The reference geometry of the aluminum alloy nozzle.

The mathematical model starts with relation between pressure and velocity of fluid, Bernoulli expression for ideal fluids on a current line is [5]:

$$
\frac{v^{2}}{2 g}+\frac{p}{\gamma}+z=\text { Const }
$$

where $v$ is fluid velocity $[\mathrm{m} / \mathrm{s}], \mathrm{p}$ is fluid pressure $[\mathrm{Pa}], \mathrm{z}$ - nozzle distance $[\mathrm{mm}], \gamma$ - specific gravity unit volume $\left[\mathrm{N} / \mathrm{m}^{3}\right]$ and $g$ is gravitational acceleration $\left[\mathrm{m} / \mathrm{s}^{2}\right]$.

At the beginning of the SolidWorks 2019 Fluid Flow Analysis, the material from which the nozzle geometry is made will be chosen. The material chosen to simulate this small-sized nozzle is an aluminum alloy 6061, which has the properties presented in Table 1 [6]:

Table 1. Properties of Aluminum 6061 Alloy.

\begin{tabular}{|l|l|l|}
\hline Property & Value & Units \\
\hline Elastic Modulus & 69000 & $\mathrm{~N} / \mathrm{mm}^{2}$ \\
\hline Shear Modulus & 26000 & $\mathrm{~N} / \mathrm{mm}^{2}$ \\
\hline Mass Density & 2700 & $\mathrm{Kg} / \mathrm{m}^{3}$ \\
\hline Tensile Strength & 124.084 & $\mathrm{~N} / \mathrm{mm}^{2}$ \\
\hline Yield Strength & 55.1485 & $\mathrm{~N} / \mathrm{mm}^{2}$ \\
\hline Thermal Expansion Coefficient & $2.4 \mathrm{e}^{-05}$ & $/ \mathrm{k}$ \\
\hline Thermal Conductivity & 170 & $\mathrm{~W} / \mathrm{m} \cdot \mathrm{K}$ \\
\hline Specific Heat & 1300 & $\mathrm{~J} / \mathrm{kg} \cdot \mathrm{k}$ \\
\hline
\end{tabular}

As it can be seen in Figure 1 the incidence angle of the hot water jet has $45^{\circ}$, at the end it's provided with a small ell so that the jet is distributed as close to the nozzle axis as possible. The temperature of the hot fluid is around $353 \mathrm{~K}$ and the cold one is $293 \mathrm{~K}$. In the analysis of SolidWorks 2019 we will study several cases, the temperature of the fluids is kept constant during the simulations, the mixing angles of the fluids will be changed. 


\section{RESULTS AND DISCUSSION}

Simple geometric elements have been chosen for this simulation, these elements being common to most of the nozzles. The way of working is the following: the CAD model was realized and then the SolidWorks 3D model of the chosen element was subjected for analysis. We study initially two cases in which the hot fluid is sent directly to the nozzle without any radius of connection at two angles $30^{\circ}$ and $45^{\circ}$. Figures 2 and 3 shows the temperature and the velocity variations along the nozzle (in the mixing area of the fluids) for the two angles of incidence [6].
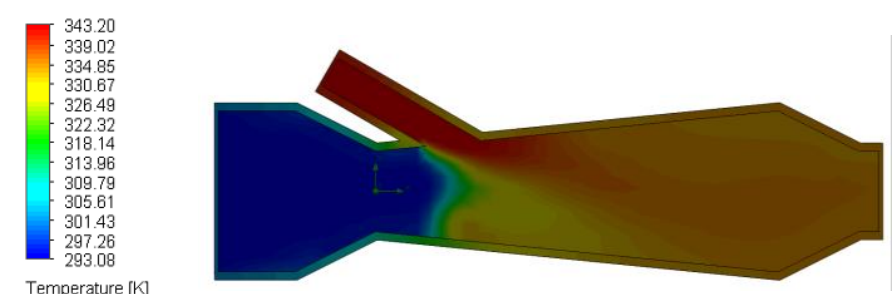

a.

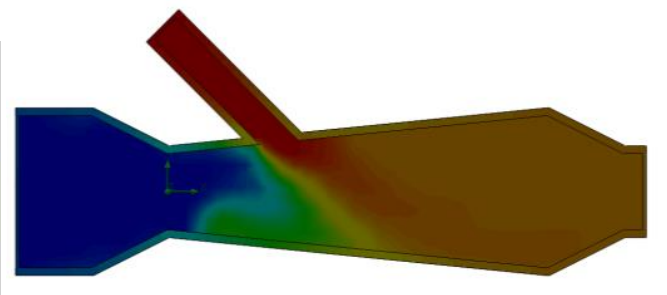

b.

Fig. 2. Temperature variation of the mixed fluids:

a. angle $30^{\circ}$; b. angle $45^{\circ}$

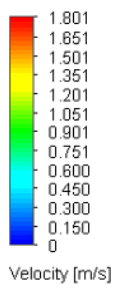

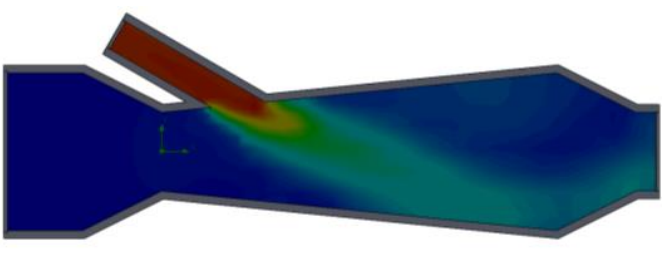

a.

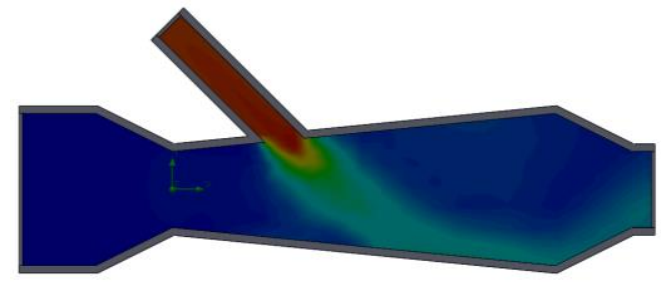

b.

Fig. 3. Velocity variation of the mixed fluids:

a. angle $30^{\circ}$; b. angle $45^{\circ}$.

For a better identification of the phenomena that appear inside the nozzle and the behavior of the fluid, and for a better visualization of these values, an extraction of them in graphs was made from the simulation carried out by the computer, Figure 4. It is important, first of all, the evolution of these values in the axis of the diaphysis, so a coordinate system was attached and number of 31 points along the $0 x$ axis were analyzed (at $1 \mathrm{~mm}$ distance from each other).

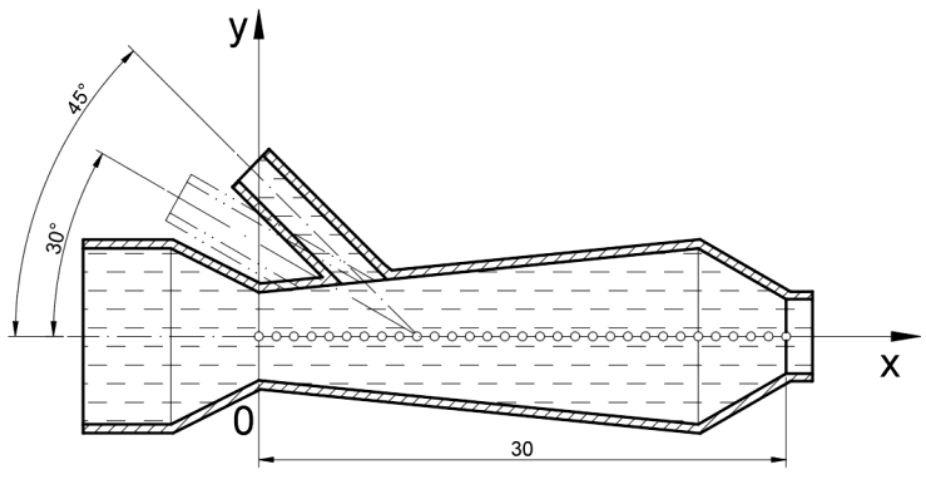

Fig. 4. Extraction mode from simulation of graphs of variation of fluid parameters.

At a first visualization of these distributions, we can observe that in the case of temperatures, a smaller angle leads to the mixing of these fluids to occur in the divergent area of the nozzle. At the $30^{\circ}$ angle the hot fluid jet is sent further to the outlet. The mixing of these two fluids is more intense and the hot jet has a rather small 
influence on the wall of the nozzle. The velocity distribution of this fluid shows us more clearly the hot fluid jet that influences more in the case of the $45^{\circ}$ angle, which leads to an additional heating of the fluid.

The graphs of variation of the parameters of the working fluid as well as a comparison of them are presented in Figure 5.

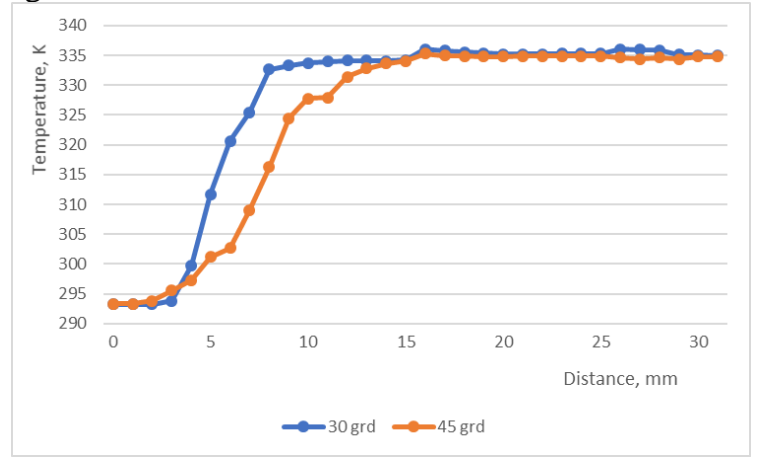

a.

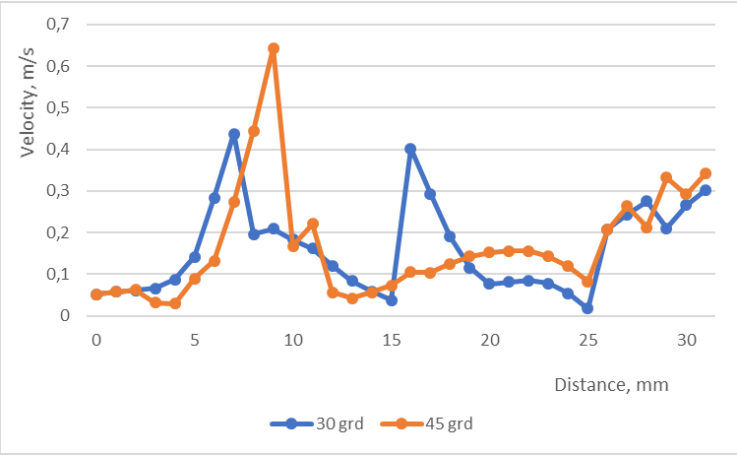

b.

Fig. 5. Parameter variation graphs for the two angles:

a. temperature; b. velocity.

Since it can be easily observed that the $30^{\circ}$ angle is more advantageous in terms of fluid flow through the nozzle, we will continue to maintain this angle and thus distribute this hot fluid as close to the axis of the nozzle. In addition, we will do an analysis in which the fluid jet is sent along the 0x axis perfectly horizontal.

If is analyzed the inflow of the hot fluid under the angle of $30^{\circ}$ we notice that it reaches the mixing temperature more quickly, and from the point of view of the variation of the velocities along the nozzle, also at the angle of $30^{\circ}$ a variation is observed in a range lower values, which may be beneficial in terms of the flow of this fluid.

The temperature and velocity distribution for the two cases is presented in Figure 6:
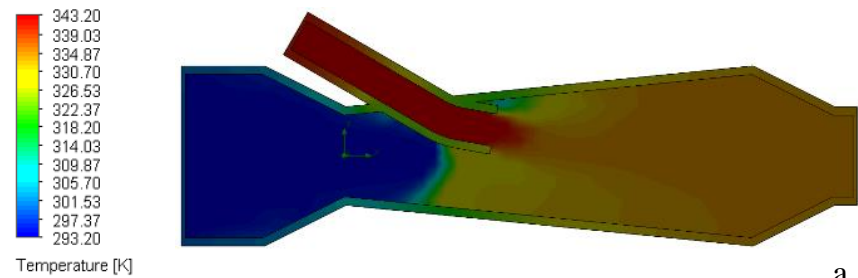

a.
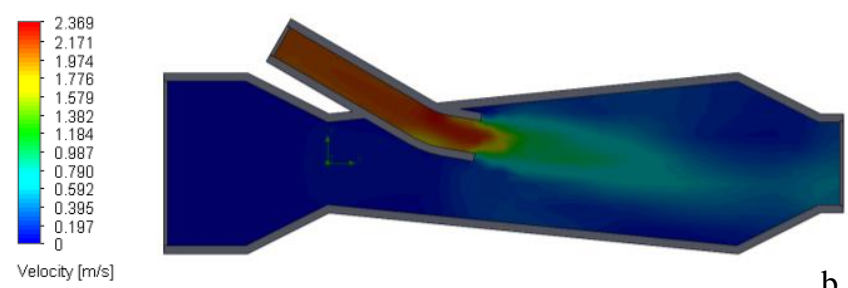

b.
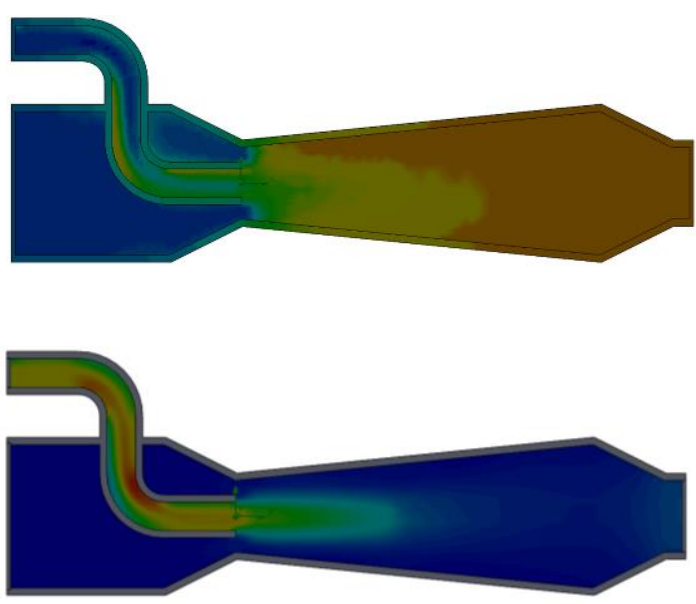

Fig. 6. Temperature and velocity distribution in the nozzle: a. angle de $30^{\circ}$; angle de $0^{\circ}$.

Although in case the hot fluid enters an angle of $0^{\circ}$ compared to the cold one it would be more advantageous in terms of the distribution of temperatures and the jet inside the nozzle, it will be considered the adoption of constructive solutions as simple as possible in order not to create additional hydraulic resistances in the fluid jet due to the pipe parts inserted in the main flow jet, of course for more demanding fluids that have higher densities. 
As in the previous cases when a free discharge of the hot fluid jet was used in the nozzle, values of the temperatures and velocities of the fluid along the same axis will be extracted, values that can be seen in the distribution diagrams presented in Figure 7:

As one can see a better distribution of temperature variation and fluid velocity along the axis of the nozzle, it's recorded if the incidence angle is as small, if it can be close to $0^{\circ}$.

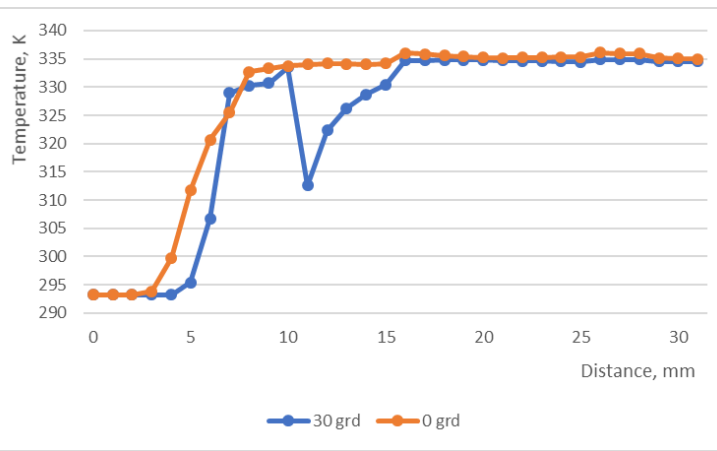

a.

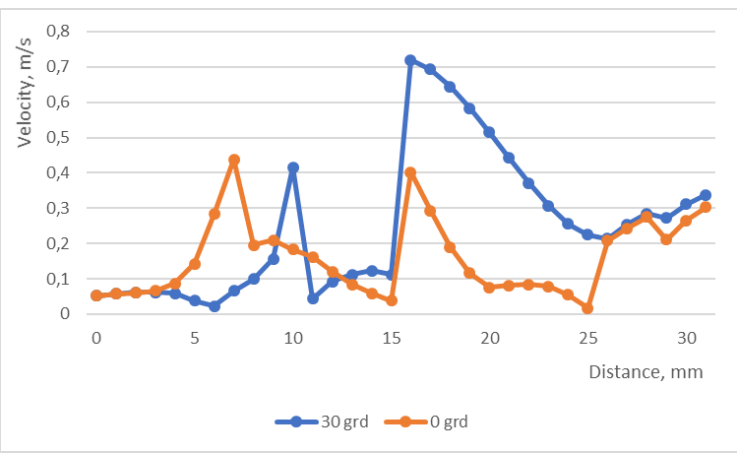

b.

Fig. 7. Parameter variation graphs for the two angles:

a. temperature; b. velocity.

In the case of the inlet nozzle of the heated agent at $30^{\circ}$, an influence of the temperature given by the thermal transfer made through the pipe inserted into the fluid jet from the nozzle is observed, the outflow temperatures for the two cases analyzed being similar. Regarding of the fluid velocity can be observed that at a small angle of entry of the hot fluid the variation is softer or in other words it falls within a narrower range of values.

This CFD analysis performed with SolidWorks 2019 can be the starting point in making fluid mixing nozzles as efficient as possible, by studying an infinite variety of geometries, all these geometries must be technologically correlated with the way they can be made. It's known that depending on the material chosen, a constructive variant can be chosen as best as possible.

The material can also influence the heat transfer inside and outside the nozzle and thus the material can be chosen according to needs. Due to the thematic conductive properties of each material it can be chosen to increase or decrease the transfer from the inside to the outside.

\section{CONCLUSIONS}

A better knowledge of the phenomena that occur in the case of mixing two fluids with different temperature is good for the most correct choice of constructive variant for heat exchangers. As can be seen from the paper, an angle as close to the general flow as possible can generate a fluid jet that influences as little as possible the walls of the nozzle.

However, there may be cases in practice when it's desired to mix these two fluid jets as quickly as possible and in which the choice of a larger angle of incidence is more appropriate. In the case of heat exchangers through the mixing of fluids, a good mixing of them must be achieved, as well as a thermal transfer as efficient as possible.

In this work through simulations for several nozzle geometries can be concluded from the analysis of flow in the nozzle is very important, following this analysis can optimize the size and shape of the nozzles so that the cooling fluid to be distributed more efficiently in the mixing area.

Compared to the situations presented in this paper, there may be cases in which there are various limitations of the nozzle geometry from the point of view of its practical realization, designing efficient mixing heat exchangers should be a compromise between the thermal efficiency of the mixture and the possibility of technical realization is achieved. 
As shown in this paper, a special importance presents the fluid depending on the geometry of the nozzle to eliminate hydraulic blockages that may arise and can lead to increased fluid pumping power and thus to a higher consumption of fluid.

\section{REFERENCES}

[1] Fatahi, R., Farzanega, A., An analysis of multiphase flow and solids separation inside Knelson Concentrator based on four-way coupling of CFD and DEM simulation methods, Minerals Engineering, vol. 126, 2018, p. 130-144.

[2] Rajeswari, M., Azizi, K., Hasim, S., Abdullah, M.K., Muhammad, A.M., Abdullah, M.Z., CFD simulation and experimental analysis of flow dynamics and grinding performance of opposed fluidized bed air jet mill, International Journal of Mineral Processing, vol. 98, no. 1-2, 2011, p. 94-105.

[3] Ramponi, R., Blocken, B., CFD simulation of cross-ventilation flow for different isolated building configurations: Validation with wind tunnel measurements and analysis of physical and numerical diffusion effects, Journal of Wind Engineering and Industrial Aerodynamics, vol. 104-106, 2012, p. 408-418.

[4] Zhong, Y., Lian, H., Deqi, C., Haidong, L., Dewen, Y.,Wenxing, L., CFD simulation on the flow and heat transfer characteristics of mist flow in wire-wrapped rod bundle, Nuclear Engineering and Design, vol. 345, 2019, p. 62-73.

[5] Olaru, I., The fluid flow simulation through to a convergent nozzle, Journal of Engineering Studies and Research, vol. 19, no. 2, 2013, p. 76-80.

[6] https://www.solidworks.com/sw/simulation-heat-transfer-confirmation.htm (20.10.2019). 